\title{
Conformational Changes in High-Density Lipoprotein Nanoparticles Induced by High Payloads of Paramagnetic Lipids
}

\author{
Pedro Ramos-Cabrer ${ }^{1,2,3^{*}}$, Francois Fay ${ }^{4}$, Brenda L. Sanchez-Gaytan ${ }^{4}$, Jun Tang ${ }^{4,5}$, José Castillo ${ }^{3}$, Zahi \\ A. Fayad ${ }^{4}$, Willem J. M. Mulder ${ }^{4}$ \\ ${ }^{1}$ Molecular Imaging Unit, ClC biomaGUNE, Paseo Miramón 182, 20009 \\ Donostia-San Sebastian, Spain \\ ${ }^{2}$ Ikerbasque, Basque Foundation for Science, Maria Diaz de Haro 3, 48011 \\ Bilbao, Spain \\ ${ }^{3}$ Clinical Neurosciences Research Laboratory, Department of Neurology, University Clinical Hospital \\ Santiago, Health Sciences Institute (IDIS), Travesa da choupana s/n, 15706 \\ Santiago de Compostela, Spain \\ ${ }^{4}$ Translational and Molecular Imaging Institute, Icahn School of Medicine at Mount Sinai, One \\ Gustave Levy Place, NY 10029 \\ New York, NY, United States of America \\ ${ }^{5}$ Radiology Department. Memorial Sloan Kettering Cancer Center, 1275 York Avenue, NY 1275 \\ New York, NY, United States of America
}

\author{
*Corresponding Author \\ Pedro Ramos Cabrer \\ Email:pramos@cicbiomagune.es \\ $\mathrm{CIC}$ biomaGUNE \\ Paseo Miramón 182 \\ 20009 Donostia-San Sebastian \\ Spain \\ Tel: (34) 943005426 \\ Fax: (34) 943005301
}




\section{SUPPORTING INFORMATION (SI)}

\section{Synthesis of HDL nanoparticles}

As we have already described in the manuscript, phospholipids; DMPC (1,2-dimyristoyl-snglycero-3-phosphocholine), MHPC (1-myristoyl-2-hydroxy-sn-glycero-3-phosphocholine) and Gd-DTPA-DSA (gadolinium diethylenetriamine pentaacetatedistearylamide) were purchased from Avanti Polar Lipids (Alabaster, AL, USA). Apolipoprotein A-1 solution (APOA1) was donated by CSL Ltd. (Parkville, Australia). Phosphate buffered saline 1x (PBS) was obtained from Fischer Scientific (Pittsburg, PA, USA).

HDL nanoparticles were prepared as described elsewhere. [1] In brief, stock solutions of 10 $\mathrm{mg} / \mathrm{ml}$ of each lipid were prepared, using a 3:1 ( $/ \mathrm{v})$ mix of chloroform and methanol as solvent, mixing proper volumes of each lipid solution for each composition tested. Total amount of lipids was fixed to $2.5 \mathrm{mg} / \mathrm{ml}$ in 5 batches or $5 \mathrm{mg} / \mathrm{ml}$ in another 5 batches, prepared for each composition. The ratio between single chained lipids (MHPC) versus double chained lipids (DMPC + Gd-DTPA-DSA) was always kept constant at $1 / 10(\mathrm{~m} / \mathrm{w})$. Eight sets of $n=10$ identical batches of particles where prepared at 8 different rations DMPC/Gd-DTPA-DSA (see Table S1). The solvent of each mixture of lipids was removed under reduced pressure to form a lipid film, which was thoroughly dried under vacuum overnight. Films were subsequently hydrated with $5 \mathrm{ml}$ of phosphate buffered saline (PBS $1 \mathrm{x}$ ) and a proper amount of ApoA1 protein (ApoA1 vs. total amount of lipids in a constant mass ratio of 1 to 2.5 ). Solutions were then incubated at $37^{\circ} \mathrm{C}$ for $3 \mathrm{~h}$, and then submitted to sonication for $60 \mathrm{~min}$ (avoiding overheating by keeping the samples on ice during the whole process). Finally samples were centrifuged $(2880 \mathrm{G}, 30 \mathrm{~min})$ to remove metal debris from the sonicator probe, and centrifuged again (2880G, $30 \mathrm{~min}$ ) in $100 \mathrm{KDa}$ MWCO tubes (Vivaspin, Sartorius Corporation, Edgewood, NY) to remove uncomplexed lipids. Samples were subsequently split in two sets and incubated overnight either at $25^{\circ} \mathrm{C}$ (bench) or 37 ${ }^{\circ} \mathrm{C}$ (incubator). Further sample storage was performed at $4{ }^{\circ} \mathrm{C}$. Eight different compositions of particles were prepared with molar fractions of gadolinium phospholipids ranging $0<x<0.33$. Ten batches of each composition were prepared giving a total of $n=80$ samples (see Table S1).

\begin{tabular}{ccccc}
\hline Particle & \multicolumn{3}{c}{ Mass ratios (w/w) } & \multirow{2}{*}{$\mathrm{X}_{\mathrm{Gd}}$} \\
\cline { 2 - 4 } name & Lipids / APOA1 & MHPC / (dc-lipids)* & DMPC / Gd-DTPA-DSA & 0 \\
\hline HDL00 & $2.5 / 1$ & $1 / 10$ & $1 / 0$ & 0.098 \\
HDL02 & $2.5 / 1$ & $1 / 10$ & $1 / 0.2$ & 0.175 \\
HDL04 & $2.5 / 1$ & $1 / 10$ & $1 / 0.4$ & 0.207 \\
HDL05 & $2.5 / 1$ & $1 / 10$ & $1 / 0.5$ & 0.237 \\
HDL06 & $2.5 / 1$ & $1 / 10$ & $1 / 0.6$ & 0.263 \\
HDL07 & $2.5 / 1$ & $1 / 10$ & $1 / 0.7$ & 0.287 \\
HDL08 & $2.5 / 1$ & $1 / 10$ & $1 / 0.8$ & 0.333 \\
HDL10 & $2.5 / 1$ & $1 / 10$ & $1 / 1$ & \\
\hline
\end{tabular}

*dc-lipids: double-chained lipids (DMPC + Gd-DTPA-DSA)

Table S1. HDL nanoparticles prepared with different fractions of gadolinium lipids as traceable MRI contrast agent. The $\mathrm{w} / \mathrm{w}$ ratio of alipoprotein A1 (APOA1) versus the total amount of lipids was kept constant at (1/2.5), and the w/w ratio of single-chained lipids (MHPC) versus double chained lipids (DMPC + Gd-DTPA-DSA) was also kept constant at (1/10), for all formulations. 


\section{Measurement and processing of MR relaxation rates}

$\mathrm{T} 1$ and T2 MR relaxation times of each individual sample were measured using a Minispec system (Bruker BioSpin MRI GmbH, Ettlingen, Germany) operating at $60 \mathrm{MHz}$. T1 values where acquired in duplicate with a saturation-recovery sequence of 12 exponentially distributed repetition times ranging $100 \mathrm{~ms}$ to $12 \mathrm{~s}$, while T2 values were acquired 4 times, using a CPMG sequence of 3600 points with an interval of tau $=1 \mathrm{~ms}$.

Calculated longitudinal $(R 1=1 / T 1)$ and transversal $(R 2=1 / T 2)$ relaxation rates for each sample were plotted versus the total concentration of lipids (in $\mathrm{mM}$ ). In Figure S1 we present the results for one batch of each of the 8 sets of HDL nanoparticles with different content in gadolinium lipids. Similar results were obtained for the other 9 batches of each set (data not shown).
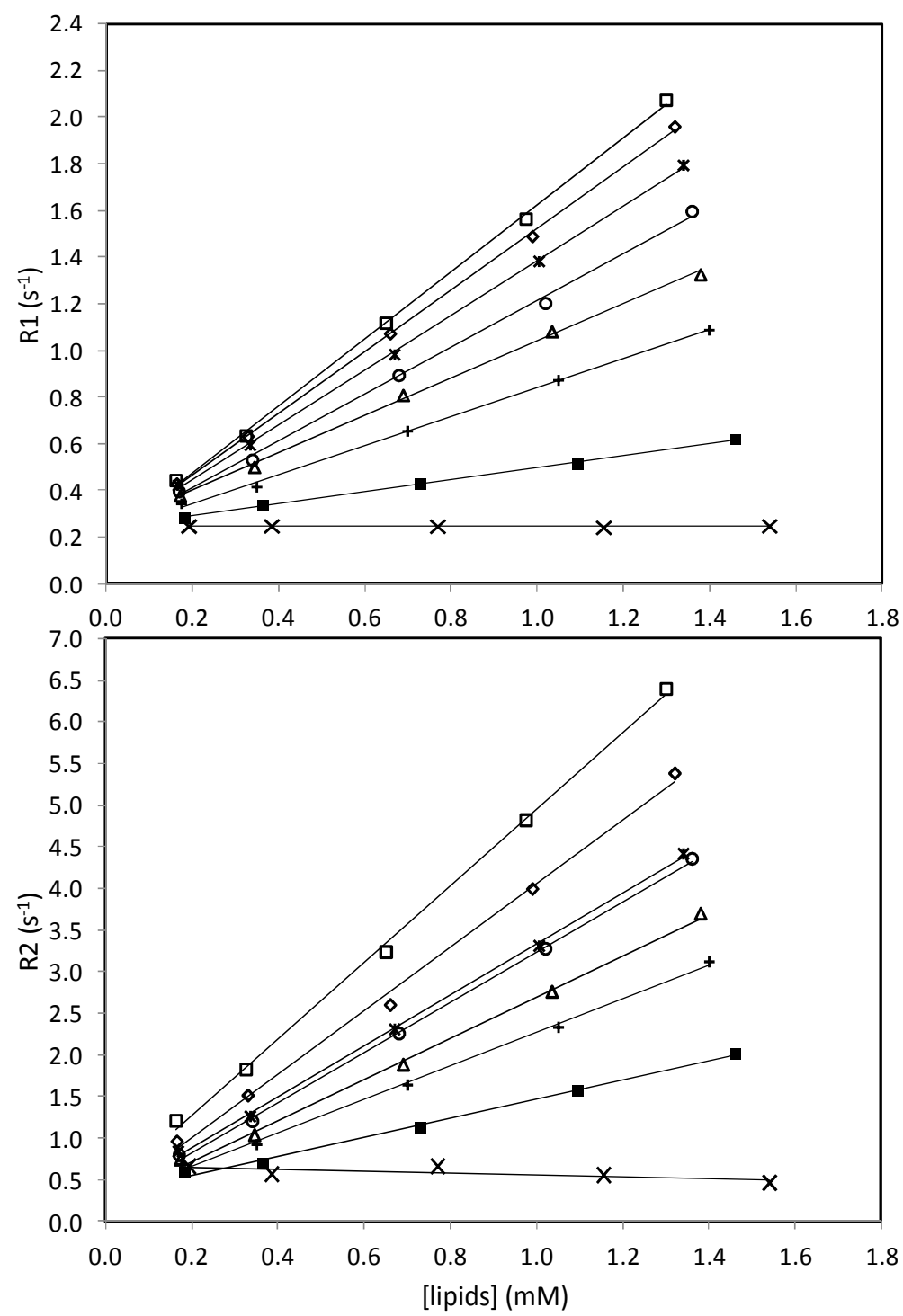

Figure S1 Longitudinal (R1) and transversal (R2) relaxation rates of 5 dilutions of HDL samples versus the total amount of lipids. Eight sets ( $n=10$ identical batches each) of HDL particles were prepared containing molar fractions of Gd-DTPA-DSA of $X_{\text {Gd-DTPA-DSA }}=0.0(\times), 0.098(\square), 0.175(+), 0.207(\triangle)$, $0.237(\bigcirc), 0.263(*), 0.287(\diamond)$ and $0.333(\square)$. Results shown here correspond to one batch of each of the 8 sets of rHDL particles, and similar results were obtained for the other 9 batches. 
$\mathrm{R} 1$ and $\mathrm{R} 2$ plots for any of the $\mathrm{n}=80$ samples were fit to linear regressions to provide the MR relavivities ( $r 1$ or $r 2$, respectively) as the slope of those fittings. Mean ( \pm standard deviation) values of $r 1$ and $r 2$ were then calculated for each of the 8 sets of $n=10$ identical batches of particles (see Figure 1 and accompanying text in the manuscript).

\section{Transmission electronic microscopy}

TEM micrographs were performed for selected samples, as described in the methods section.
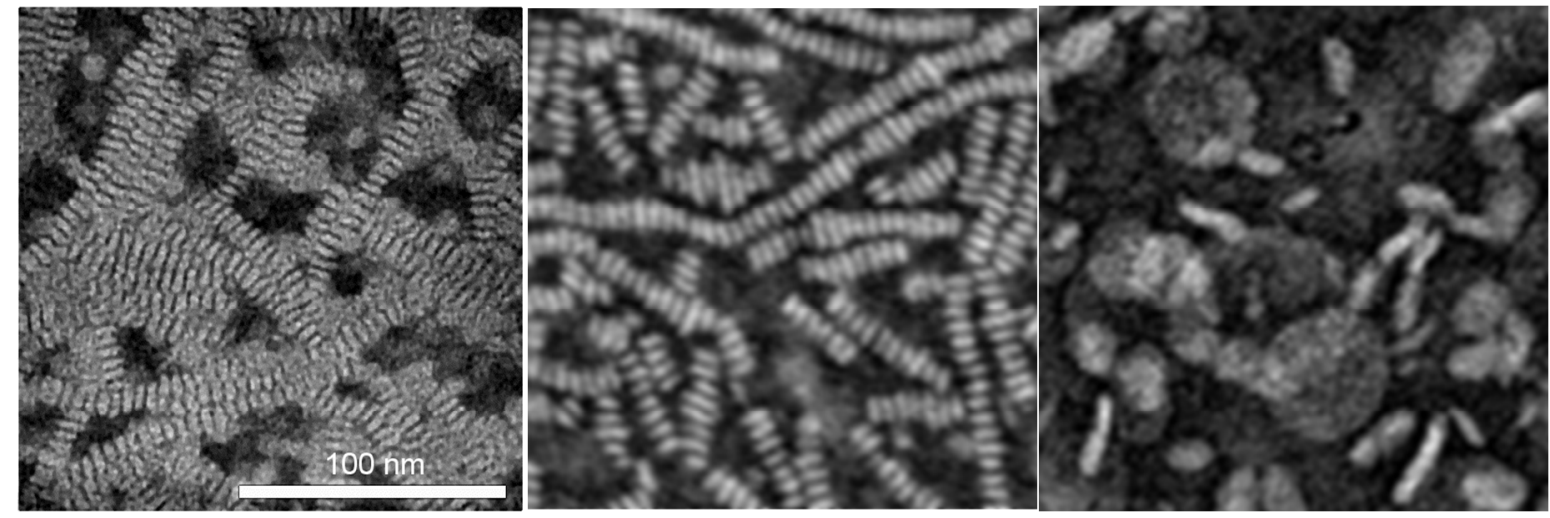

Figure S2. Transmission Electronic Microscopy micrograph of; left) a sample of naive HDL particles, showing the typical pilled-disks structures characteristic for this molecule. Middle) a sample of HDL with a low molar fraction of gadolinium lipids $(x=0.1)$, showing similar conformation, and right) a sample of HDL with a high molar fraction of gadolinium lipids $(x=0.33)$, showing the formation of vesicles instead of disks. Scale bar is the same for all the images.

\section{Modeling of experimental data}

The analysis of data presented in the manuscript, and in particular data related to the particle size and shape (Figures 2 and 3 in the manuscript and figure S2) point to the following facts (summarized in the scheme presented below):

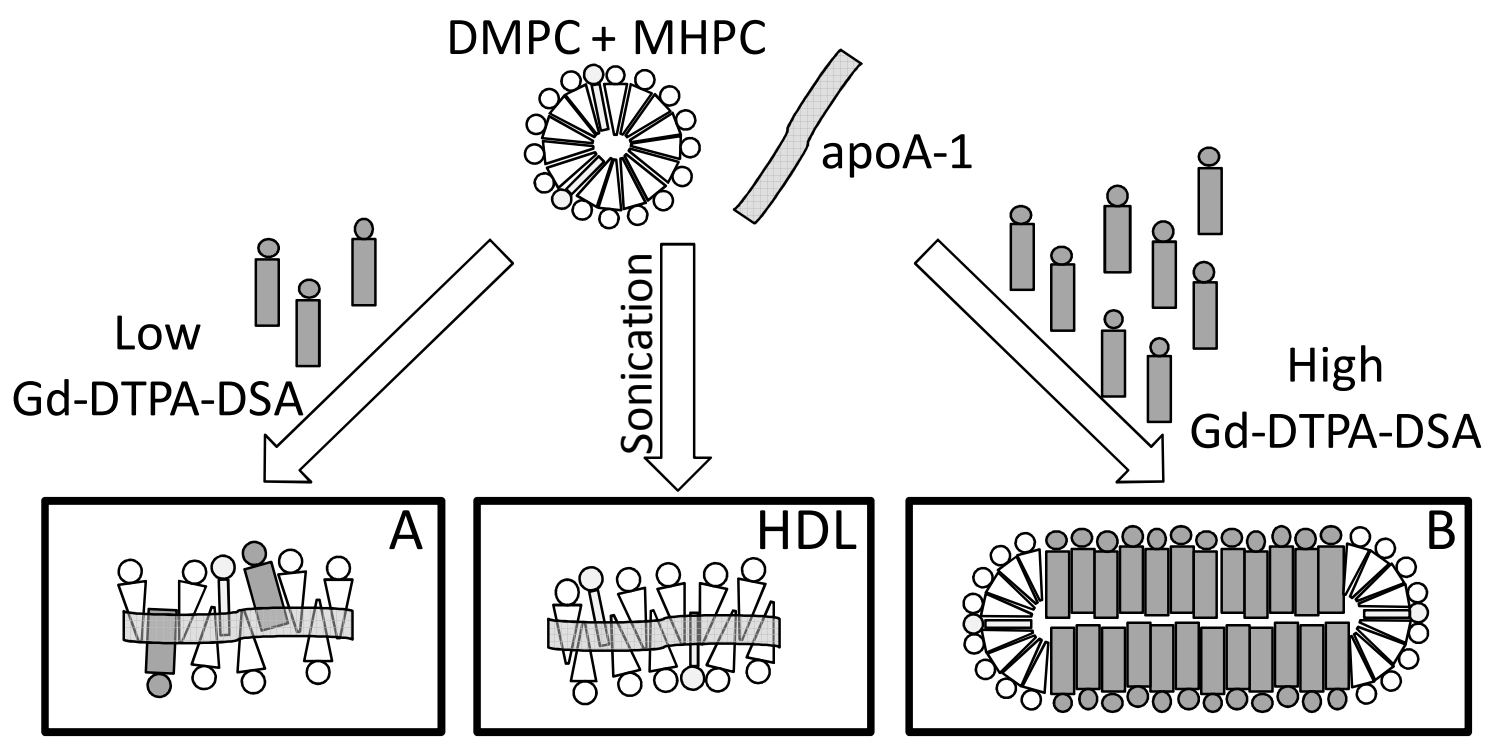


Figure S3 Schematic representation of the model. Under sonication (energy), a mix of DMPC, MHPC and APOA1 in solution yields HDL particles. Low amounts of Gd-DTPA-DSA lipids can be packed in disk-like conformations (A). Above a certain threshold, alternative molecular rearrangements (B particles) are more stable. The large aggregate shown here is an example for purely descriptive purposes and the factual structure may be different.

1) naïve HDL nanoparticles present a typical disk-like structure of circa $8 \mathrm{~nm}$ of diameter.

2) The substitution of a small fraction of the double chained lipid DMPC by the Gadolinium labeled double-chained phospholipid Gd-DTPA-DSA equally yields in the formation of HDL disk-like particles (named A).

3) The use of higher amounts of gadolinium lipids favors the formation of a different type of structures (named B) with a six-fold larger size than HDL disks. (The large aggregate shown here is an example for purely descriptive purposes and the factual structure may be different.)

The final statement may be justified by higher steric hindrance expected for the packaging of the gadolinium lipid used in this work, as compared to DMPC, according to their structures:

DMPC:

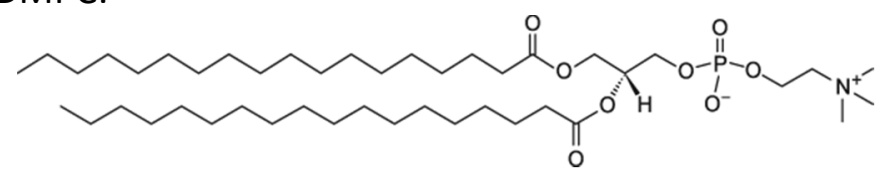

\section{Gd-DTPA-DSA}

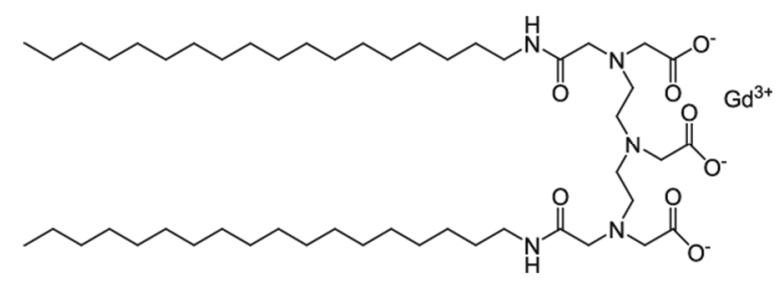

4) It seems that there is a threshold or critical value for the molar fraction of Gd-DTPA-DSA for this transition. Below the critical value only type $A$ particles were observed. Above this critical value, either $A$ or $B$ type particles are obtained during synthesis, but once formed no transition between $A$ and $B$ structural arrangements has been observed (a process with presumably high activation energy in both directions, according to Fukuda et al. [2, 3[ The proposition of the existence of this threshold is based on the experimental data. Thus, we first used the size values measured for any of the $n=80$ samples prepared (plotted in Figure 2 in the manuscript) to calculate the fraction of batches of type $B$ particles $\left(X_{B}\right)$ obtained at each molar fraction of gadolinium used: 
Table S2. Fraction of particles of type A and B depending on lipids composition of HDL.

\begin{tabular}{|c|c|c|c|}
\hline $\begin{array}{c}\text { molar fraction } \\
\text { of gadolinium } \\
\left(\mathrm{X}_{\mathrm{Gd}}\right)\end{array}$ & $\begin{array}{c}\text { NA: Number of } \\
\text { measured } \\
\text { batches } \\
\text { with } \mathrm{d} \sim 8.2 \mathrm{~nm} \\
(\text { Type } \mathrm{A})\end{array}$ & $\begin{array}{c}\text { NB: Number of } \\
\text { measured } \\
\text { batches } \\
\text { with } \sim 51.7 \mathrm{~nm} \\
(\text { Type } \mathrm{B})\end{array}$ & $\begin{array}{c}\text { Fraction of Type } \\
\text { B particles }\end{array}$ \\
\hline 0.000 & 20 & 0 & 0 \\
\hline 0.098 & 21 & 0 & 0 \\
\hline 0.175 & 27 & 3 & 0,100 \\
\hline 0.207 & 22 & 6 & 0,214 \\
\hline 0.237 & 19 & 10 & 0,345 \\
\hline 0.263 & 11 & 21 & 0,656 \\
\hline 0.287 & 6 & 24 & 0,800 \\
\hline 0.330 & 10 & 22 & 0,687 \\
\hline
\end{tabular}

The calculated parameter $\left(x_{B}\right)$ seems to be directly linearly correlated to the molar fraction of gadolinium lipids in the particle:

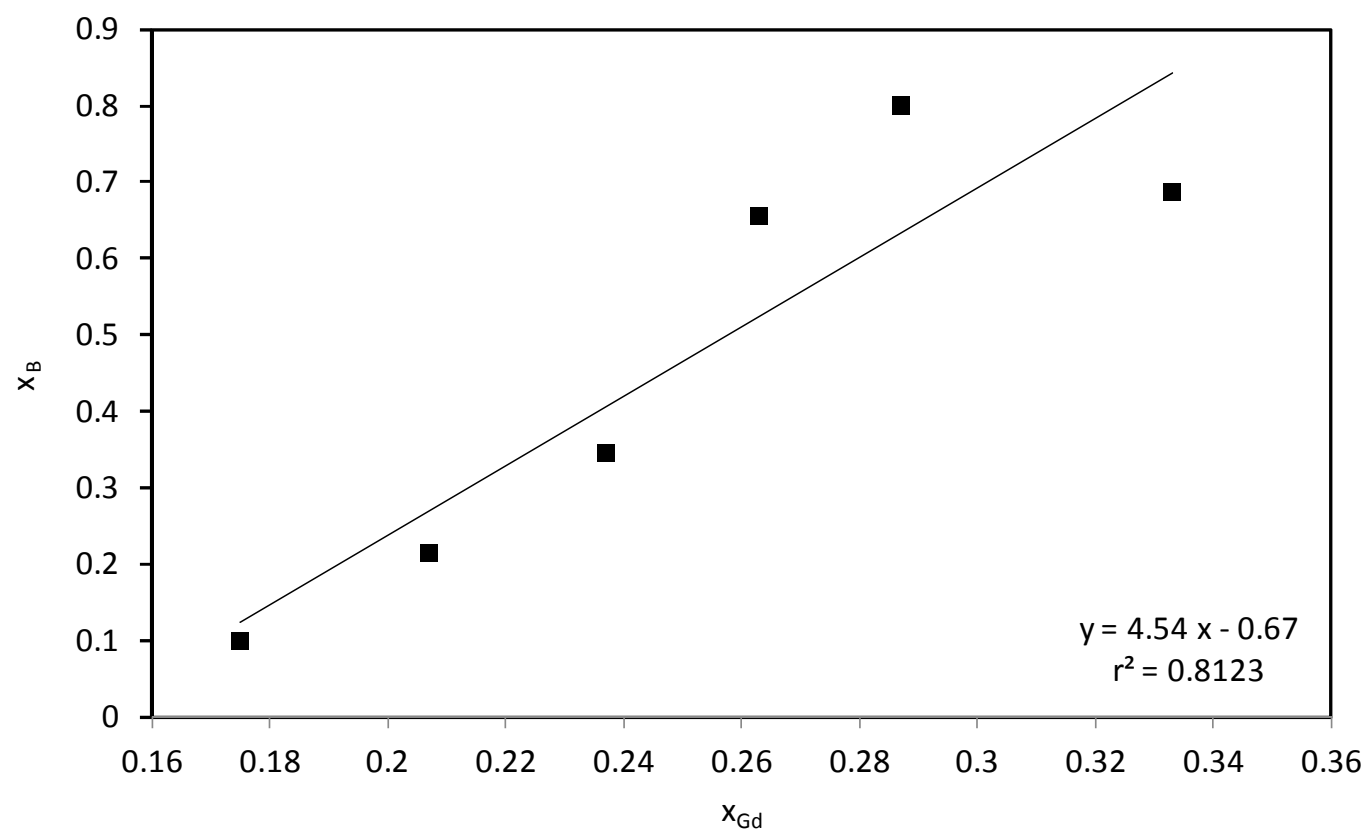

Figure. S4. Representation of the fraction of Type B particles in solution vs. the molar fraction of gadolinium lipids used for synthesis, as presented in Table S2.

Fitting of the data provides the following linear equation:

$X_{B}=4.54 X_{G d}-0.67$

According to prior assumptions of our model, when a set of $n$ batches of particles is synthesized using a high load of gadolinium lipids, some of them will correspond to type $A$ particles, with a size of $d_{H}(A)=8.2 \pm 1.6 \mathrm{~nm}$, while the rest would be type $B$ particles, with a size of $d_{H}(B)=51.7 \pm 7.3 \mathrm{~nm}$. At this point we are going to consider the mean size of a set of 
$n$ batches of particles as a linear combination of $d_{H}(A)$ and $d_{H}(B)$, weighted by the fraction of samples of each kind obtained for the whole set of batches:

$\mathrm{d}_{H}($ mean n samples $)=\left[8.2\left(1-X_{B}\right)+51.7\left(X_{B}\right)\right] n m$

Thus, combining equations (1) and (2) we obtain an equation that should predict the experimental behavior of data presented in Figure 2 of the manuscript and reproduced below:

$d_{H}($ mean $)=197.49 X_{G d}-21.0$

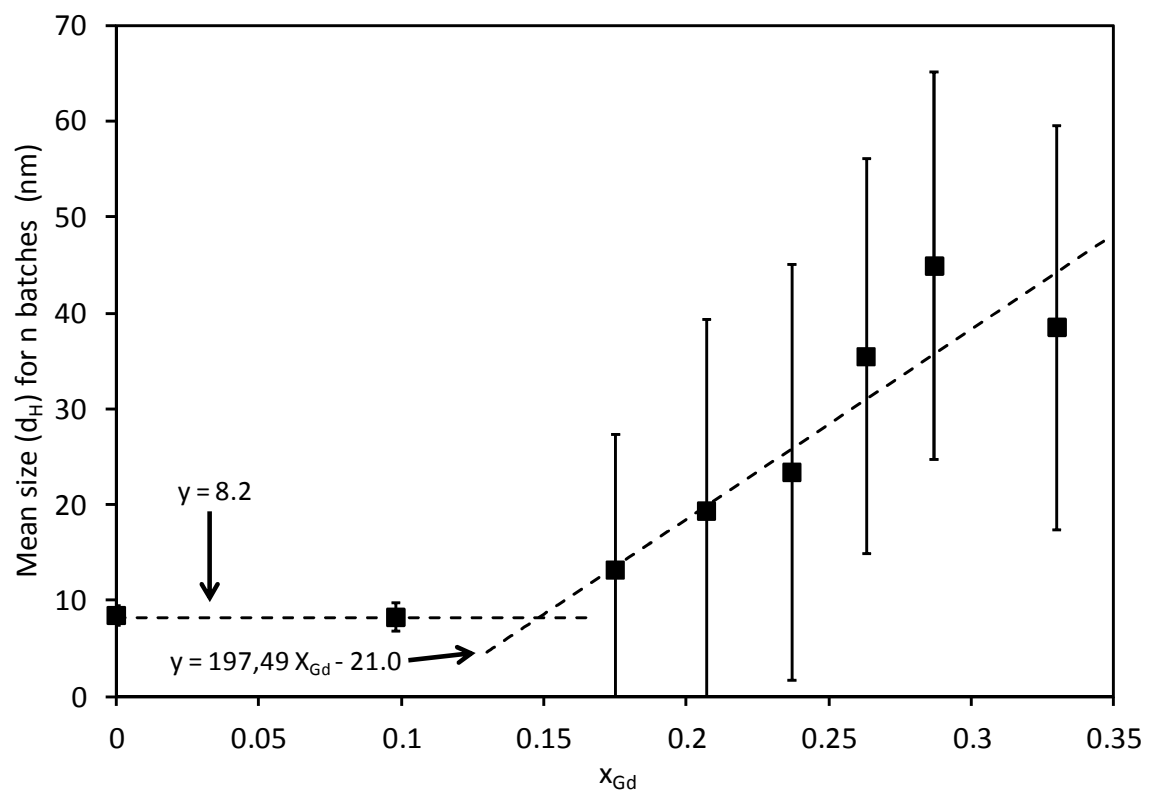

Figure S5. Plot of the mean ( \pm SD) sizes experimentally obtained for each of the 8 sets of $n=10$ batches of particles synthesized vs. the molar fraction of gadolinium lipids used for synthesis (filled squares). The straight dashed line is the representation of equation (3), and the horizontal dashed line corresponds to $y=d_{H}(A)=8.2 \mathrm{~nm}$.

As we can see in Figure S5, the proposed model explains the behavior of the system respect to the mean values obtained for the size of the particles as a function of the molar fraction of gadolinium lipids, and confirms the existence of a critical molar fraction of gadolinium lipids that can be used for the synthesis of HDL disk-like nanoparticles (defined by the crossing of the two dashed lines). The use of amounts of gadolinium lipids above this threshold during the synthesis would lead to the obtaining of a certain proportion of batches of particles that will not correspond to HDL disk-like structures but to larger aggregates. The frequency at which this event would take place increases linearly with the molar fraction of gadolinium used for the synthesis.

In the particular case of Gd-DTPA-BSA the critical molar fraction of gadolinium lipid that we could use to ensure that $100 \%$ of the prepared batches would maintain the typical HDL disk-like structure (crossing of dashed lines in Figure S5) is achieved at: 


\section{Critical $\mathrm{X}_{\mathrm{Gd}-\mathrm{DTPA}-\mathrm{DSA}}=\mathbf{0 . 1 4 8}$}

One should expect that this threshold varies with the nature and structure of the gadolinium lipid used for the labeling of HDL nanoparticles.

Like it happened for particle sizes we propose that particles $A$ and $B$ are species with different $M R$ relaxivity values (i.e. $r 1(A)$ and $r 1(B) ; r 2(A)$ and $r 2(B)$ ) and that the mean of $r 1$ or $r 2$ values measured for a set of $n$ batches of particles is a linear combination of these individual values, weighted by the fraction of samples of each kind present in the whole set.

Under these circumstances the mean $\mathrm{r} 1$ and $\mathrm{r} 2$ values can be calculated as:

$r 1$ (mean n samples) $=\left[r 1(A)\left(1-X_{B}\right)+r 1(B)\left(X_{B}\right)\right] m^{-1} s^{-1}$

$r 2$ (mean n samples) $=\left[r 2(A)\left(1-X_{B}\right)+r 2(B)\left(X_{B}\right)\right] m^{-1} s^{-1}$

or, rearranging terms, as:

$r 1($ mean n samples $)=r 1(A)+[r 1(B)-r 1(A)]\left(X_{B}\right)$

$r 2($ mean n samples $)=r 2(A)+[r 2(B)-r 2(A)]\left(X_{B}\right)$

According to these expressions, if we plot the experimental mean values of $r 1$ and $r 2$ versus the fraction of B-type particles (as calculated in Table S2), for values $\mathrm{X}_{\mathrm{Gd}}>0.148$ (the regime where type $B$ particles exist) we should obtain linear relationships for both parameters:

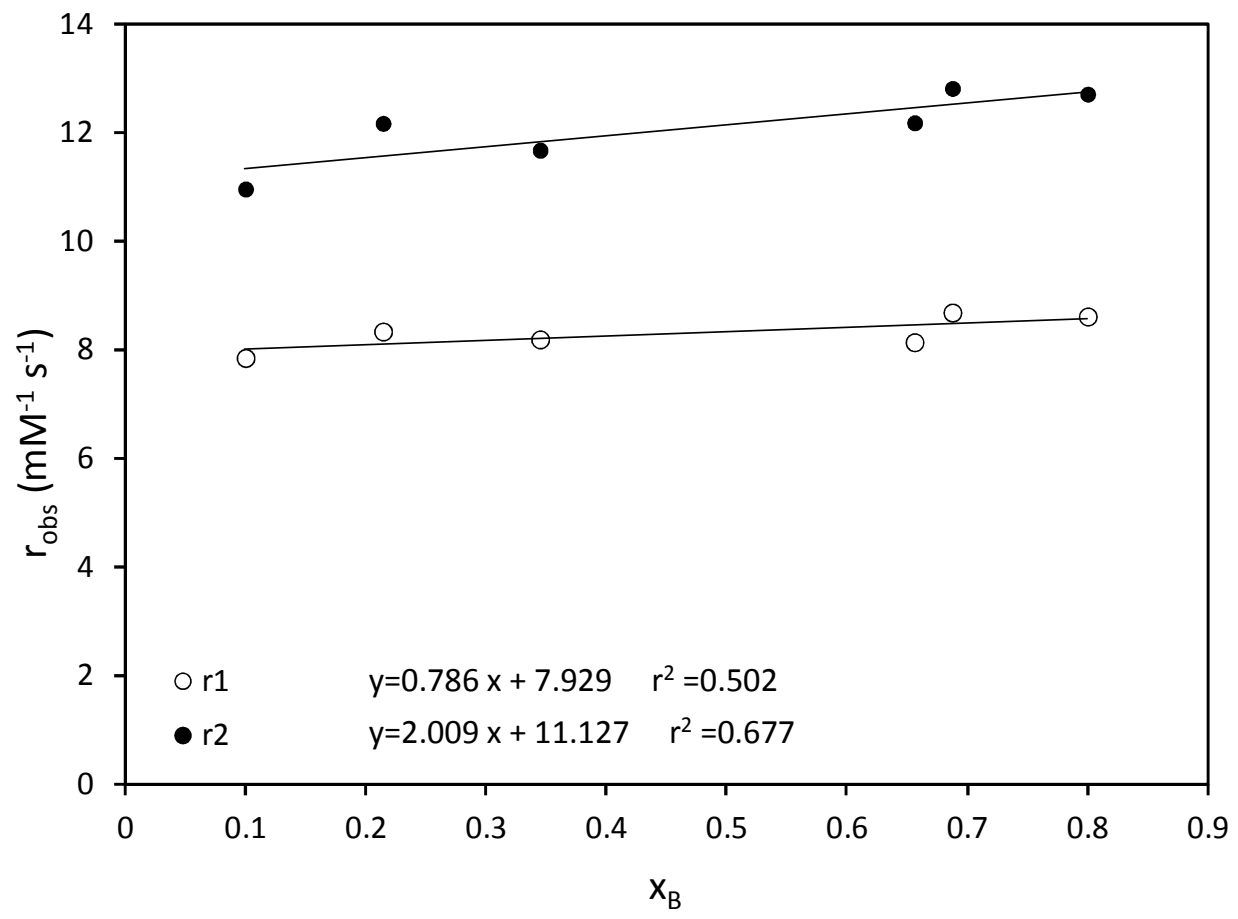


Figure S6. Observed longitudinal $(r 1)$ and transversal $(r 2)$ ionic relaxivities observed for the synthesized particles vs. the fraction of B-type particles presented in solution (calculated in table S2).

Using the intercepts and slopes of the linear fittings of this plot in equations (6) and (7), we obtained the individual MR relaxivities of particles A and B:

$$
\begin{array}{lll}
r 1(A)=7.93 \mathrm{mM}^{-1} \mathrm{~s}^{-1} & ; & r 1(B)=8.72 \mathrm{mM}^{-1} \mathrm{~s}^{-1} \\
r 2(A)=11.13 \mathrm{mM}^{-1} \mathrm{~s}^{-1} ; & r 2(B)=13.14 \mathrm{mM}^{-1} \mathrm{~s}^{-1}
\end{array}
$$

Using these values and combining equations (1), (6) and (7) we obtain two expressions that predict the experimental behavior of the data, including the apparent formation of a plateau for MR relaxivities (Figure S7):

$\mathrm{r} 1$ (mean $\mathrm{n}$ samples) $=7.40+3.52 \mathrm{X}_{\mathrm{Gd}}$

$\mathrm{r} 2$ (mean n samples) $=9.76+9.12 \mathrm{X}_{\mathrm{Gd}}$

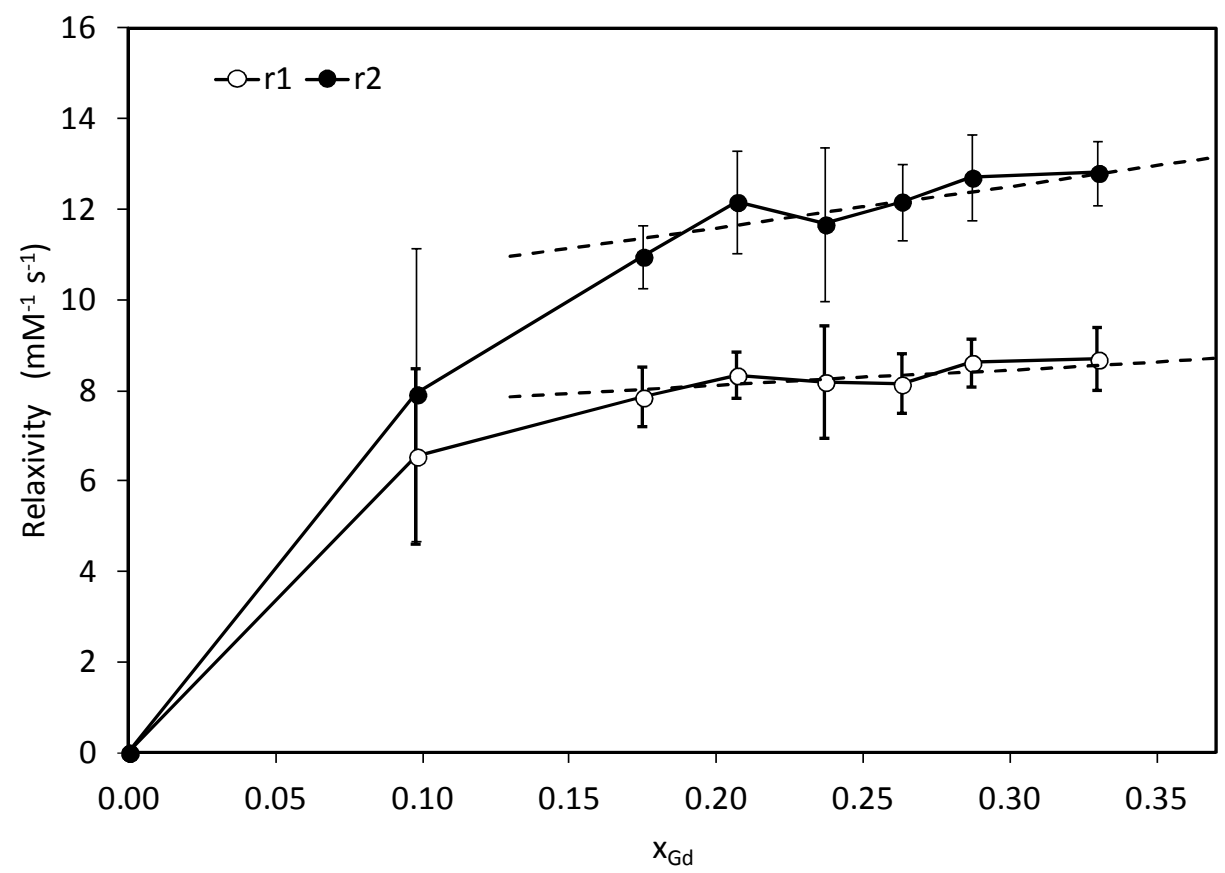

Figure S7. Variation of the longitudinal ( $r 1$, hollow circles) and transversal ( $r 2$, filled circles) MR relaxivities for HDL formulations versus their content in Gd-lipids (expressed as molar fraction). Dashed lines correspond to equations (8) and (9).

Dashed lines in the figure correspond to equations (8) and (9), hollow and solid circles correspond to experimental mean values of $r 1$ and $r 2$, respectively, and the dotted lines were calculated to cross the plots of equation (8) and (9) at $X_{\text {Gd-DTPA-DSA }}=0.148$ (the critical values calculated for DLS data) and considering that $r 1$ and $r 2$ of naïve $\mathrm{HDL}$ are both equal to cero (experimentally corroborated). These lines are potentially compatible with the existence of the same critical value for the molar fraction of gadolinium lipids predicted 
before. However, experimental data acquired in this regime of molar fractions is not enough to confirm this fact.

In summary, we have developed a predictable model that explains the behavior of both, the mean particle size and the mean ionic relaxivity, of sets of $n$ batches of HDL nanoparticles prepared at different molar fractions of gadolinium lipids for the MR labeling of the particles.

We propose the existence of a critical value for the molar fraction of gadolinium laden lipids that can be used for the labeling of HDL nanoparticles. Above this critical value, the combination of lipids and APOA1 molecules yields either the typical HDL disk-like nanopartículas or a different (larger) kind of particles. The probability of obtaining large particles growths linearly with the molar fraction of gadolinium lipids added. The critical value of $X_{G d}$ most likely depends on the nature and properties of the chosen lipids.

\section{References}

1 R. Duivenvoorden, J. Tang, D. P. Cormode, A. J. Mieszawska, D. Izquierdo-Garcia, C. Ozcan, M. J. Otten, N. Zaidi, M. E. Lobatto, S. M. van Rijs, B. Priem, E. L. Kuan, C. Martel, B. Hewing, H. Sager, M. Nahrendorf, G. J. Randolph, E. S. Stroes, V. Fuster, E. A. Fisher, Z. A. Fayad and W.J. Mulder, A statin-loaded reconstituted high-density lipoprotein nanoparticle inhibits atherosclerotic plaque inflammation. Nat. Commun., 2014, 5, 3065

2 M. Fukuda, M. Nakano, M. Miyazaki and T. J. Handa, Thermodynamic and Kinetic Stability of Discoidal High-Density Lipoprotein Formation from Phosphatidylcholine/Apolipoprotein A-I Mixture. Phys. Chem. B, 2010, 114, 8228

3 M. Nakano, M. Fukuda, T. Kudo, M. Miyazaki, Y. Wada, N. Matsuzaki, H. Endo and T. Handa, Static and Dynamic Properties of Phospholipid Bilayer Nanodiscs. J. Am. Chem. Soc., 2009, 131, 8308 